\title{
What Factors are Associated With 90-day Episode-of-care Payments for Younger Patients With Total Joint Arthroplasty?
}

\author{
Shweta Pathak PhD (1), Cecilia M. Ganduglia MD, DrPH, Samir S. Awad MD, MPH, \\ Wenyaw Chan PhD, John M. Swint PhD, Robert O. Morgan PhD
}

Received: 7 March 2017/ Accepted: 6 July 2017/Published online: 13 July 2017

(C) The Association of Bone and Joint Surgeons \& 2017

\begin{abstract}
Background Total joint arthroplasty (TJA) has been identified as a procedure with substantial variations in inpatient and postacute care payments. Most studies in this area have focused primarily on the Medicare population and rarely have characterized the younger commercially insured populations. Understanding the inpatient and postdischarge care service-component differences across 90-day episodes of care and factors associated with payments for younger patients is crucial for successful
\end{abstract}

Each author certifies that neither he or she, nor any member of his or her immediate family, has funding or commercial associations (consultancies, stock ownership, equity interest, patent/licensing arrangements, etc) that might pose a conflict of interest in connection with the submitted article.

All ICMJE Conflict of Interest Forms for authors and Clinical Orthopaedics and Related Research ${ }^{\circledR}$ editors and board members are on file with the publication and can be viewed on request.

The University of Texas Health Science Center at Houston approved the human protocol for this investigation, and each author certifies that all investigations were conducted in conformity with ethical principles of research.

This work was performed at The School of Public Health, University of Texas Health Science Center (Houston, TX, USA).

Electronic supplementary material The online version of this article (doi:10.1007/s11999-017-5444-0) contains supplementary material, which is available to authorized users.

S. Pathak ( $ه)$, C. M. Ganduglia, W. Chan, J. M. Swint,

R. O. Morgan

School of Public Health, University of Texas Health Science

Center, 1200 Herman Pressler Blvd., Houston, TX 77030, USA

e-mail: shweta.pathak@uth.tmc.edu

S. S. Awad

Operative Care Line, Surgery Service, Michael E. DeBakey VA

Medical Center, Houston, TX, USA implementation of bundled payments in TJA in nonMedicare populations.

Purpose (1) To assess the mean total payment for a 90day primary TJA episode, including the proportion attributable to postdischarge care, and (2) to evaluate the role of procedure, patient, and hospital-level factors associated with 90-day episode-of-care payments in a nonMedicare patient population younger than 65 years.

Method Claims data for 2008 to 2013 from Blue Cross Blue Shield of Texas were obtained for primary TJAs. A total of 11,131 procedures were examined by aggregating payments for the index hospital stay and any postacute care including rehabilitation services and unplanned readmissions during the 90-day postdischarge followup period. A three-level hierarchical model was developed to determine procedure-, patient-, and hospital-level factors associated with 90-day episode-of-care payments.

Results The mean total payment for a 90-day episode for TJA was USD 47,700 adjusted to 2013 USD. Only 14\% of 90-day episode payments in our population was attributable to postdischarge-care services, which is substantially lower than the percentage estimated in the Medicare population. A prolonged length of stay (rate ratio [RR], 1.19; 95\% CI, 1.15-1.23; $\mathrm{p} \leq 0.001$ ), any 90-day unplanned readmission $(\mathrm{RR}, 1.64 ; 95 \% \mathrm{CI}, 1.57-1.71 ; \mathrm{p} \leq$ $0.001)$, computer-assisted surgery (RR, $1.031 ; 95 \% \mathrm{CI}$,

S. S. Awad

Department of Surgery, Baylor College of Medicine, Houston, TX, USA

J. M. Swint

The Center for Clinical Research and Evidence-Based Medicine,

McGovern Medical School at The University of Texas Health

Science Center at Houston, Fannin, Houston, TX, USA 
1.004-1.059; $\mathrm{p} \leq 0.05)$, initial home discharge with home health component (RR, 1.029; 95\% CI, 1.013-1.046; $\mathrm{p} \leq$ 0.001 ), and very high patient morbidity burden (RR, 1.105; 95\% CI, 1.062-1.150; $\mathrm{p} \leq 0.001)$ were associated with increased TJA payments. Hospital-level factors associated with higher payments included urban location (RR, 1.29; 95\% CI, 1.17-1.42; $\mathrm{p} \leq 0.001$ ), lower hospital case mix based on average relative diagnosis related group weight (RR, 0.94; 95\% CI, 0.89-0.95; p $\leq$ 0.001), and large hospital size as defined by total discharge volume (RR, 1.082; 95\% CI, 1.009-1.161; $\mathrm{p} \leq 0.05)$. All procedure, patient, and hospital characterizing factors together explained $11 \%$ of variation among hospitals and $49 \%$ of variation among patients.

Conclusion Inpatient care contributed to a much larger proportion of total payments for 90-day care episodes for primary TJA in our younger than 65-year-old commercially insured population. Thus, inpatient care will continue to be an essential target for cost-containment and delivery strategies. A high percentage of hospital-level variation in episode payments remained unexplained by hospital characteristics in our study, suggesting system inefficiencies that could be suitable for bundling. However, replication of this study among other commercial payers in other parts of the country will allow for conclusions that are more robust and generalizable.

Level of Evidence Level II, economic analysis.

\section{Introduction}

TKA and THA are two of the most common and costly inpatient surgical procedures performed on Medicare beneficiaries [8]. However, the demand for joint arthroplasties is increasing among patients younger than 65 years because of younger patients seeking better mobility and less pain attributable to arthritis [19, 31].

Total joint arthroplasty (TJA) has been identified as a procedure with substantial variations in inpatient and postacute care costs [3, 10, 28, 33, 39]. Consequently, several private and public payers have been testing alternative payment models in an effort to reduce costs, improve value, and emphasize quality in patients' transition from surgery to recovery [14, 16, 40]. For example, The Centers for Medicare and Medicaid Services (CMS) implemented its first mandatory bundled payment model for joint replacement care in April 2016 [8]. Under this type of payment model, participant hospitals receive a single payment from Medicare for any care related to a TJA episode that begins with an admission to a participant hospital and ends 90 days after discharge. There are potential cost savings for hospitals that successfully align incentives, but there is a simultaneous risk associated with costs exceeding the negotiated target if hospitals fail in that goal.

Policies designed for Medicare also may have important effects on prices negotiated between nongovernmental payers and hospitals. As such, various bundled payments for TJA are increasing among private payers, such as insurance providers and employers [14, 16, 40]. Consequently, hospitals considering participating in such initiatives and involved in the TJA care cycle have sought to evaluate their specific service-component cost differences across each TJA episode of care and factors associated with episode payments [5, 10, 17].

Given the high variability in inpatient and postdischarge payments among TJA episodes of care and the rising incidence of TJA in the younger non-Medicare population, we sought (1) to assess the mean total payments for a 90day primary TJA episode, including the proportion attributable to postdischarge care, and (2) to evaluate the role of procedure, patient, and hospital-level factors associated with 90-day episode-of-care payments in a nonMedicare patient population younger than 65 years.

\section{Methods}

We obtained a population-based cohort from Blue Cross Blue Shield of Texas enrollment and claims data for 2008 to 2013. Our dataset included payments for all claims for medical care and ancillary services (facility and professional) filed with Blue Cross Blue Shield of Texas and a member enrollment file for Blue Cross Blue Shield of Texas members from 2008 to 2013. Indicators of race and poverty levels of enrollee residential zip codes were obtained through the American Community Survey (ACS) data in lieu of patient-level indicators [36]. The classification of urban and rural zip codes was based on the United States Department of Agriculture Economic Research Service Nonurban-Urban Continuum Codes [38].

We received approval for performing this study from our institutional review board. Our analysis cohort consists of all Blue Cross Blue Shield of Texas members younger than 65 years enrolled in preferred hospital organizations (PPO and $\mathrm{PPO}+$ ) or point of service plans (POS) who underwent an elective, primary total TJA (this includes unicompartmental, bicompartmental, and tricompartmental knee procedures and total hip replacement procedures as defined by ICD-9 Procedure Codes 81.51 and 81.54) between July 1, 2008 and October 1, 2013. Each member had a minimum enrollment in a PPO or POS plan for 9 months. We used patient diagnoses codes over the 6-month preindex admission period for a TJA (knee or hip) to 
calculate patient-morbidity burden using patient diagnoses codes. Patient-morbidity burden (categories ranging from low morbidity to very high morbidity) was assigned using The Johns Hopkins Adjusted Clinical Groups ${ }^{\mathbb{R}}\left(\mathrm{ACG}^{\mathbb{R}}\right)$ Risk Adjustment System [18]. The morbidity-burden calculation assigns a higher value to conditions that are expected to have higher consumption of healthcare resources (based on condition severity, duration, need for specialty care, diagnostic certainty, and type of etiology) and thus represents a patient's resource-use burden. The "index" hospital stay for analysis was the date of hospital admission for a THA or TKA through the date of discharge. Thus, we excluded joint surgeries performed in outpatient settings as indicated by the service-type code (inpatient versus outpatient) in our dataset (Fig. 1). The 90day episode of care refers to the index stay and a 90-day postdischarge followup period. To ensure that the unit of analysis is a TJA episode and the observation period for each TJA episode was a total of 9 months, we excluded patients with more than one TJA during a 9-month interval. We only included patients younger than 65 years at the end of the postdischarge 90-day followup to exclude those who might qualify for Medicare (Fig. 1). We only included patients with diagnosis-related group (DRG) codes for major joint replacement or reattachment of lower extremity (469 or 470), because these codes have been found to be
Fig. 1 The inclusion and exclusion criteria for calculation of 90-day episode-of-care costs are shown. DRG = diagnosis related group.

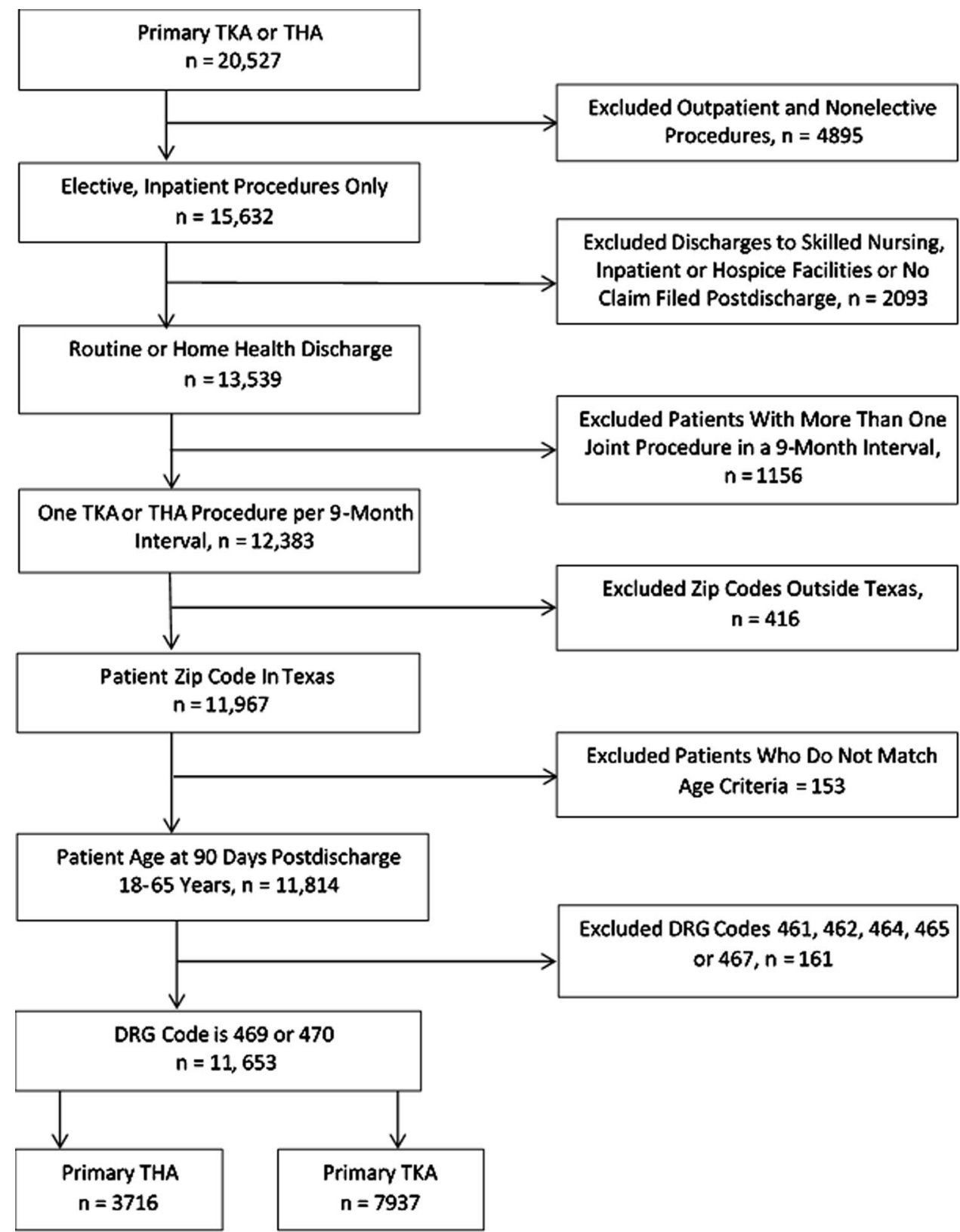


most conducive to bundling and are the only ones included in the Comprehensive Care for Joint Replacement program guidelines published by Medicare [8]. Our sample had less than $1 \%$ of those with a DRG of 469 (major joint replacement or reattachment of lower extremity with Major Complication/Comorbidity). As per Medicare guidelines [8], we included all facility and professional services during the 90-day followup period including all unplanned readmissions to an inpatient, acute care facility but excluded any planned readmissions (eg, staged bilateral procedures).

We identified 20,527 TJAs between 2008 and 2013. After applying our inclusion and exclusion criteria (Fig. 1), our study cohort consisted of 11,653 TJAs from 242 different Texas hospital hospitals. Of the 11,653 episodes that met the inclusion criteria, 453 had missing service hospital information including zip codes and 69 had missing information for the patient economic status indicator. Since these constituted only $0.04 \%$ of our total observations, we removed them from our analyses. Thus, our final sample had 11,131 TJAs from 242 hospitals. Of the 11,131 total episodes, approximately $5 \%(\mathrm{n}=512)$ were primary TJAs repeated on the same patients.

The primary outcome of interest for our study was the total dollar amount associated with a 90-day TJA episode. We calculated the total amount by aggregating payments associated with the index hospital stay and included professional or facility claims that were triggered in the 90-day postdischarge period. All payments are amounts rendered to providers by enrolled patients and Blue Cross Blue Shield of Texas based on contractual agreements. All payments were adjusted to 2013 USD using the medical component of the Consumer Price Index. Independent variables used in our model included procedure-characterizing factors such as inpatient length of stay, type of joint involvement (hip or knee), the use of computer-assisted surgery, discharge status (home health or routine), and whether there was any subsequent, unplanned readmission during the 90-day postdischarge period. Other factors included patient characteristics such as age at the end of 90-day episode, sex, and patient-morbidity burden.

Economic status and race were measured at the patients' residential zip codes, which included the percent of families below the poverty line as a proxy for socioeconomic status and the percent of the population which is nonwhite [36]. Such area-based indicators have been considered useful in risk-adjustment studies and for monitoring disparities in healthcare systems [2, 9, 15]. Both socialcontext variables were split in four categories using quartiles. We included the zip code distance between patients' residential zip code and hospital location as an indicator of geographic access to care.
For hospital characteristics, we constructed a binary variable to indicate whether the hospital was in an urban area, and calculated the number of total discharges during one calendar year for each inpatient hospital as a proxy for hospital size. We calculated the hospital case mix, which is the average relative DRG weight of a hospital, to adjust for differences in the severity of cases across annual hospital discharges. Thus, the hospital size and case-mix variables were calculated using Blue Cross Blue Shield of Texas claims data.

\section{Statistical Analysis}

Analyses were conducted using STATA ${ }^{\circledR}$, Version 13.0 (Stata Corp, College Station, TX, USA). Factors of interest were summarized using percentages and frequencies for categorical variables and means and SDs for continuous variables. Comparisons of patient and hospital characteristics between THAs and TKAs were made with chi-square tests for categorical variables and Mann-Whitney U tests for continuous variables.

Our dataset consisted of TJA episodes that were naturally nested in patients, with patients nested in hospitals. We used the generalized linear mixed model with the gamma distribution, logarithmic link function, and robust standard errors to model the right-skewed payments in our data $[11,13,20,21]$. We estimated a series of five models (Supplemental Table 1. Supplemental material is available with the online version of $C O R R^{\circledR}$.) to understand how each set of procedure, patient, and hospital characteristics separately contributed to the payment variation among patients and variation among hospitals in our data. The first was an unconditional/null model with no patient, provider, or hospital factors. The second model included procedurecharacterizing factors only, the third model included patient-level factors only, the fourth included hospital-level factors only, while the fifth model was the all-factor model with procedure, patient, and provider factors included simultaneously. The five models allowed us to calculate the proportional changes in the variance components for each model using the null model as the reference [29]. The allfactor model allowed us to estimate how each factor was associated with the mean payment for 90-day care episodes.

Using gamma regression analysis, we calculated the rate ratio (RR), which is the rate increase in the mean payment per episode for a discrete change in each procedure, patient, and hospital factor in our model. Corresponding marginal effects were calculated to determine the estimated changes in dollar amounts per discrete change in patient, procedure, and hospital factors based on the fitted model. 
Table 1. Descriptive statistics for all TJAs

\begin{tabular}{|c|c|c|c|c|c|c|c|}
\hline \multirow[t]{2}{*}{ Patient and procedure characteristics } & \multicolumn{2}{|c|}{ All TJAs $(\mathrm{n}=11,131)$} & \multicolumn{2}{|c|}{ THAs $(\mathrm{n}=3543)$} & \multicolumn{2}{|c|}{ TKAs $(\mathrm{n}=7588)$} & \multirow[b]{2}{*}{$\mathrm{p}$ Value } \\
\hline & $\%$ & Number & $\%$ & Number & $\%$ & Number & \\
\hline Female (versus male) & 55.27 & 6152 & 48.80 & 1729 & 58.3 & 4423 & $\leq 0.001$ \\
\hline Age & & & & & & & $\leq 0.001$ \\
\hline Younger than 56 years & 33.86 & 3769 & 42.20 & 1495 & 30 & 2274 & \\
\hline 56 to 60 years & 31.13 & 3465 & 28.80 & 1021 & 32.2 & 2444 & \\
\hline 61 to 65 years & 35.01 & 3897 & 29.00 & 1027 & 37.8 & 2870 & \\
\hline Percent nonwhite* & & & & & & & 0.096 \\
\hline Quartile1 $(<0.02)$ & 24.98 & 2781 & 23.60 & 835 & 25.6 & 1946 & \\
\hline Quartile $2(0.02-0.06)$ & 24.94 & 2776 & 25.30 & 897 & 24.8 & 1879 & \\
\hline Quartile 3 (0.07-0.13) & 24.91 & 2773 & 25.90 & 916 & 24.5 & 1857 & \\
\hline Quartile $4(>0.13)$ & 25.16 & 2801 & 25.30 & 895 & 25.1 & 1906 & \\
\hline Percent below FPL* & & & & & & & $\leq 0.001$ \\
\hline Quartile $1(<0.08)$ & 24.96 & 2778 & 27.70 & 981 & 23.7 & 1797 & \\
\hline Quartile $2(0.08-0.13)$ & 24.66 & 2745 & 25.30 & 897 & 24.4 & 1848 & \\
\hline Quartile 3 (0.14-0.19) & 25.19 & 2804 & 24.20 & 856 & 25.7 & 1948 & \\
\hline Quartile 4 (>0.19) & 25.19 & 2804 & 22.80 & 809 & 26.3 & 1995 & \\
\hline Distance to hospital (miles) & & & & & & & $\leq 0.001$ \\
\hline Short (0 to 15$)$ & 50.97 & 5674 & 49.22 & 1744 & 51.79 & 3930 & \\
\hline Medium (16 to 75 ) & 38.83 & 4322 & 38.81 & 1375 & 38.84 & 2947 & \\
\hline Long $(>75)$ & 10.2 & 1135 & 11.97 & 424 & 9.37 & 711 & \\
\hline Morbidity burden & & & & & & & $\leq 0.001$ \\
\hline Low $(=2)$ & 14.03 & 1562 & 12.60 & 445 & 14.7 & 1117 & \\
\hline Moderate $(=3)$ & 70.66 & 7865 & 67.50 & 2391 & 72.1 & 5474 & \\
\hline High $(=4)$ & 12.4 & 1380 & 16.00 & 568 & 10.7 & 812 & \\
\hline Very high (= 5) & 2.91 & 324 & 3.90 & 139 & 2.4 & 185 & \\
\hline Computer-assisted surgery & 6.61 & 736 & 3.30 & 117 & 8.2 & 619 & $\leq 0.001$ \\
\hline Any 90 -day readmission & 5.38 & 599 & 5.50 & 194 & 5.3 & 405 & 0.763 \\
\hline Home health (versus routine) discharge & 54.7 & 6089 & 53.30 & 1890 & 55.3 & 4199 & 0.049 \\
\hline Length of stay (days) & & & & & & & $\leq 0.001$ \\
\hline Short (1 to 2) & 27.96 & 3112 & 35.00 & 1240 & 24.67 & 1872 & \\
\hline Medium (3 to 4 ) & 67.34 & 7496 & 60.49 & 2143 & 70.55 & 5353 & \\
\hline Long $(>4)$ & 4.7 & 523 & 4.52 & 160 & 4.78 & 363 & \\
\hline \multicolumn{8}{|l|}{ Hospital characteristics } \\
\hline Urban (versus nonurban) & 93.57 & 10,415 & 95.30 & 3377 & 92.8 & 7038 & $\leq 0.001$ \\
\hline Hospital case mix & & & & & & & 0.640 \\
\hline Low $(<0.1)$ & 33.32 & 3709 & 32.70 & 1160 & 33.6 & 2549 & \\
\hline Moderate $(0.1-$ to 0.6$)$ & 33.34 & 3711 & 33.80 & 1198 & 33.1 & 2513 & \\
\hline High $(>0.6)$ & 33.34 & 3711 & 33.40 & 1185 & 33.3 & 2526 & \\
\hline Hospital size (discharges) & & & & & & & $\leq 0.001$ \\
\hline Small $(<3,000)$ & 33.23 & 3699 & 28.30 & 1004 & 35.5 & 2695 & \\
\hline Medium (3000 to 6000 ) & 33.38 & 3715 & 34.60 & 1227 & 32.8 & 2488 & \\
\hline Large $(>6000)$ & 33.39 & 3717 & 37.00 & 1312 & 31.7 & 2405 & \\
\hline
\end{tabular}

*Patient zip code-based variables; TJA = total joint arthroplasty; FPL = Federal Poverty Level.

We ran our analysis after removing outlier payments (the highest $1 \%$ ) and found no meaningful change in our conclusions. Thus, we did not exclude any payments based on outlier values in our analysis. Since there was evidence of baseline differences $(\mathrm{p} \leq 0.05)$ between knee and hip procedures among several factors in our data (Table 1), we tested for interaction effects between type of joint procedure and other factors in our model such as computer- 
assisted surgery and discharge status. We found no meaningful interaction effects on payments for type of joint involvement and other factors in our sample.

\section{Results}

Mean Payments for 90-day Care Episode

After adjusting for the Consumer Price Index, the mean cost for a 90-day episode was USD 49,222 \pm USD 23,152 $($ mean \pm SD) for THA and USD 46,988 \pm USD 20,177 for TKA (Table 2). Only $11.5 \%$ and $14.8 \%$ of total episode payments were attributable to postdischarge care for THA and TKA, respectively.

Factors Associated With Increased Payments

The set of procedure-characterizing factors alone explained $44 \%$ variation in payments among patients but $0 \%$ variation among hospitals (Model 2, Table 3). The length of stay, computer-assisted surgery, any unplanned readmission, and an initial home health discharge were procedure factors associated with increased estimated payments (Table 4), but the type of joint involved (hip versus knee) was not associated with payments in our all-factor model. A RR of 1.19 for length of stay greater than 4 days (Table 4) indicates that the mean per-episode payment was 1.19 times higher than the length of stay of 1 to 2 days (RR, $1.19 ; 95 \%$ CI, $1.15-1.23 ; \mathrm{p} \leq 0.001)$. This corresponds to an estimated difference of USD 7943 (95\% CI, USD 6089USD 9797; $p \leq 0.001)$ independent of other factors in the model (Table 4). Similarly, any unplanned readmission during the 90-day followup added approximately USD 27,000 (95\% CI, USD 24,129-USD 30,347; $\mathrm{p} \leq 0.001$ ), and computer-assisted surgery added an additional estimated payment of USD 1200 (95\% CI, USD 152-USD 2580; $p \leq 0.05$ ) to the mean episode payment.

Patient-characterizing factors alone explained only 5\% of patient-level variation and $1 \%$ of hospital-level variation (Model 3, Table 3). Except for patient-morbidity burden, which was strongly associated with increased mean payments, area-based race and economic status indicator and distance to hospital were patient factors that were not associated with payments in our all-factor model (Table 4). A very-high morbidity burden was associated with 1.11 times higher mean payments (RR, 1.11; 95\% CI, 1.062 $1.150 ; \mathrm{p} \leq 0.001)$ than low patient-morbidity burden. This corresponded to a difference of USD 4529 (95\% CI, USD 2664-USD 6394; $\mathrm{p} \leq$ 0.001).

Hospital-characterizing factors alone (Model 4, Table 3) explained $10 \%$ of the hospital-level variation but only $3 \%$ of patient-level variation. Hospital location in an urban setting and larger hospital size were hospital factors associated with increased estimated payments while hospital case mix was associated with lower marginal payments (Table 4). Any hospital located in an urban area was associated with 1.29 times higher mean payments (RR, $1.29 ; 95 \% \mathrm{CI}, 1.17-1.42 ; \mathrm{p} \leq 0.001)$ than a rural area hospital. Conversely, hospitals with higher case mix were associated with 0.94 times lower mean payments than low

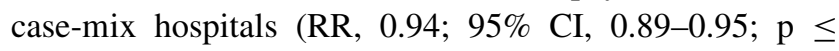
0.05 ) for 90-day episodes.

Overall, all factors (Model 5, Table 3) together explained only $11 \%$ of variation among hospitals and $49 \%$ of variation among patients, with procedure factors contributing most to payment variation among patients and hospital factors contributing most to payment variation among hospitals. The estimated mean payments for hospitals (Fig. 2) based on the fitted model showed mean payments ranging from USD 25,000 to USD 120,000 .

Table 2. Payments for 90-day episodes for TJA including THA and TKA

\begin{tabular}{|c|c|c|c|c|c|c|c|c|c|c|}
\hline \multirow[t]{2}{*}{ Variable } & \multicolumn{3}{|c|}{ Overall TJA $(\mathrm{n}=11,131)$} & \multicolumn{3}{|c|}{ THA $(\mathrm{n}=3543)$} & \multicolumn{3}{|c|}{ TKA $(\mathrm{n}=7548)$} & \multirow[b]{2}{*}{$\mathrm{p}$ Value } \\
\hline & Mean & SD & $95 \% \mathrm{CI}$ & Mean & SD & $95 \% \mathrm{CI}$ & Mean & SD & $95 \% \mathrm{CI}$ & \\
\hline \multicolumn{11}{|c|}{ Unadjusted payments (in USD) } \\
\hline Index stay & 32,042 & 12,134 & $31,817-32,268$ & 34,015 & 12,617 & $33,599-34,430$ & 31,121 & 11,790 & $30,856-31,387$ & $\leq 0.001$ \\
\hline Postdischarge & 5131 & 10,089 & $4944-5319$ & 4414 & 11,136 & $4048-4781$ & 5466 & 9543 & $5251-5681$ & $\leq 0.001$ \\
\hline Total 90-day & 37,173 & 16,122 & $36,874-37,473$ & 38,429 & 17,315 & $37,859-39,000$ & 36,587 & 15,499 & $36,238-36,936$ & $\leq 0.001$ \\
\hline \multicolumn{11}{|c|}{$\begin{array}{l}2013 \text { CPI adjusted } \\
\text { payments (in USD) }\end{array}$} \\
\hline Index stay & 41,121 & 16,116 & $40,821-41,421$ & 43,546 & 16,957 & $42,988-44,105$ & 39,989 & 15,581 & $39,638-40,340$ & $\leq 0.001$ \\
\hline Postdischarge & 6577 & 12,771 & $6341-6815$ & 5676 & 14,376 & $5202-6150$ & 6999 & 11,925 & $6731-7267$ & $\leq 0.001$ \\
\hline Total 90-day & 47,699 & 21,194 & $40,821-41,421$ & 49,222 & 23,152 & $48,460-49,985$ & 46,988 & 20,177 & $46,534-47,442$ & $\leq 0.001$ \\
\hline
\end{tabular}

$\mathrm{TJA}=$ total joint arthroplasty; $\mathrm{CPI}=$ Consumer Price Index. 
Table 3. Variance components (random-effects) of 90-day episode costs by multilevel gamma regression analysis

\begin{tabular}{|c|c|c|c|c|c|}
\hline Variable & $\begin{array}{l}\text { Model } 1 \text { (Null/no } \\
\text { factors) }\end{array}$ & $\begin{array}{l}\text { Model } 2 \text { (Procedure } \\
\text { factors only) }\end{array}$ & $\begin{array}{l}\text { Model } 3 \text { (Patient } \\
\text { factors only) }\end{array}$ & $\begin{array}{l}\text { Model } 4 \text { (Hospital } \\
\text { factors only) }\end{array}$ & $\begin{array}{l}\text { Model } 5 \\
\text { (All factors) }\end{array}$ \\
\hline $\begin{array}{l}\text { Hospital-level variance } \\
\quad \text { (SE) }\end{array}$ & $0.1013^{*}(0.0080)$ & $0.1013^{*}(0.0075)$ & $0.1005^{\star}(0.0078)$ & $0.0910^{*}(0.0076)$ & $0.0902^{\star}(0.0047)$ \\
\hline $\begin{array}{l}\text { Proportional change in } \\
\text { variance }\end{array}$ & Reference & $0.0 \%$ & $0.79 \%$ & $10.17 \%$ & $10.96 \%$ \\
\hline $\begin{array}{l}\text { Patient-level variance } \\
\text { (SE) }\end{array}$ & $0.0388^{*}(0.0048)$ & $0.0217^{\star}(0.0047)$ & $0.0371^{\ddagger}(0.0048)$ & $0.0378^{*}(0.0048)$ & $0.020^{\star}(0.0047)$ \\
\hline $\begin{array}{l}\text { Proportional change in } \\
\text { variance }\end{array}$ & Reference & $43.59 \%$ & $5.13 \%$ & $3.08 \%$ & $48.72 \%$ \\
\hline
\end{tabular}

${ }^{*} \mathrm{p}<0.001 ; \mathrm{SE}=$ standard error.

\section{Discussion}

The number of joint arthroplasties performed annually has been increasing in Medicare and non-Medicare populations and this trend is expected to continue as more people with osteoarthritis demand better quality of life and mobility [8, 19, 31]. With the CMS-mandated 90-day bundledpayment model going into effect recently in more than 800 hospitals, many payers are considering using similar payment structures for joint replacement procedures. Our study aggregated inpatient payments and followup payments for 90 days for patients with an initial home discharge after TJA in a younger, commercially insured population. We found that the inpatient component of payments contributed a considerably greater proportion of total payments for TJA compared with the postdischarge-care component. Additionally, several procedure-, patient-, and hospitalcharacterizing factors were associated with higher total 90day-episode payments and high variation in payments for TJA. These were factors such as the inpatient length of stay, computer-assisted surgery, any unplanned readmission, an initial home discharge with home-health component, patient-morbidity burden, hospital location in an urban setting, larger hospital size, and lower hospital case mix.

Most limitations in our study arise owing to the use of administrative data based on insurance claims. We modeled joint arthroplasty payments with an assumption that reimbursed payments were reflective of actual costs incurred by hospitals. Thus, the gap between actual costs and total reimbursements is not captured by our study. Claims data also are subject to coding errors or incomplete capture of patient and clinical data. We addressed this limitation by using patient-morbidity burden, which is an indicator of patient severity that we calculated using diagnoses codes during a 6-month period for a morecomprehensive capture of patient comorbidities. Moreover, we used patient residential zip code-based indicators of race and economic status to supplement our dataset with additional demographic and socioeconomic information. We were unable to obtain additional hospital characteristics owing to payer-provider contractual limitations, and approximately $89 \%$ of hospital-level variation remained unexplained in our analysis. Therefore, we cannot make a definitive conclusion whether the variability not explained by hospital characteristics was outside the scope of hospital provider influence. We also were unable to account for how insurance reimbursement structures influenced payments to hospitals.

However, because episode-based payments were not introduced among commercial carriers in Texas during the study period examined, the factors affecting total payments and the care-component differences are still relevant in the context of commercial payers that are not participating in episode-based bundled payments in joint procedures. Furthermore, our data represent approximately $25 \%$ to $30 \%$ of the commercially insured population in Texas and should be generalizable to other commercial payers serving younger Texans. Although the generalizability of our results can be influenced by regional differences in payment structures, hospital contractual factors, and patient demographics, we believe that the overall conclusions regarding inpatient and postdischarge care-component differences among younger patients and high variation in payments among hospitals are generalizable to other commercial payers serving similar populations in other parts of the country. Nevertheless, replication of this study among other non-Medicare populations will allow for conclusions that are more robust and generalizable.

The mean payments associated with an inpatient stay (USD 41,100) and a 90-day episode of care (USD 47,700) for TJA in our population are consistent with payments reported in other studies during the same period that have reported TJA payments using commercial claims data $[3,25]$. Although a previous estimate of postdischarge-care payments for Medicare showed that it can constitute up to $45 \%$ of total episode-of-care payments [22], we saw only $14 \%$ of episode payments attributable to postdischarge 
Table 4. Observed rate ratios and marginal effects for the all-factor model

\begin{tabular}{|c|c|c|c|c|}
\hline Variable & Rate ratio & $95 \% \mathrm{CI}$ & Marginal effects (in USD) & $95 \% \mathrm{CI}$ \\
\hline \multicolumn{5}{|c|}{ TJA procedure characteristics } \\
\hline \multicolumn{5}{|l|}{ Length of stay (days) } \\
\hline 1 to 2 & Ref & & & \\
\hline 3 to 4 & $1.061^{*}$ & $(1.042-1.080)$ & $2541.26^{\ddagger}$ & (1779 to 3304$)$ \\
\hline Greater than 4 & $1.190^{*}$ & $(1.147-1.234)$ & $7942.84^{*}$ & (6089 to 9797$)$ \\
\hline \multicolumn{5}{|l|}{ Any 90 -day readmission } \\
\hline No & Ref & & & \\
\hline Yes & $1.641^{*}$ & $(1.574-1.711)$ & $27,237.70^{\ddagger}$ & $(24,129$ to 30,347$)$ \\
\hline \multicolumn{5}{|l|}{ Discharge status } \\
\hline Routine & Ref & & & \\
\hline Home health & $1.029^{\ddagger}$ & $(1.013-1.046)$ & $1268.14^{\ddagger}$ & (579 to 1957 ) \\
\hline \multicolumn{5}{|l|}{ Type of TJA } \\
\hline Hip & Ref & & & \\
\hline Knee & 1.009 & $(0.990-1.028)$ & 397.66 & $(-414$ to 1209$)$ \\
\hline \multicolumn{5}{|l|}{ Computer-assisted surgery } \\
\hline No & Ref & & & \\
\hline Yes & $1.031^{*}$ & $(1.004-1.059)$ & $1366.23 *$ & (152 to 2580$)$ \\
\hline \multicolumn{5}{|l|}{ Patient characteristics } \\
\hline \multicolumn{5}{|l|}{ Age } \\
\hline Younger than 56 years & Ref & & & \\
\hline 56 to 60 years & 0.991 & $(0.980-1.001)$ & -406.32 & $(-880$ to 67$)$ \\
\hline 61 to 65 years & 0.989 & $(0.976-1.002)$ & -492.50 & $(-1061$ to 76$)$ \\
\hline \multicolumn{5}{|l|}{ Gender } \\
\hline Female & Ref & & & \\
\hline Male & 0.998 & $(0.987-1.009)$ & -95.78 & $(-574$ to 382$)$ \\
\hline \multicolumn{5}{|l|}{ Morbidity burden } \\
\hline Low $(=2)$ & Ref & & & \\
\hline Moderate $(=3)$ & $1.017^{*}$ & $(1.003-1.031)$ & $725.12 *$ & (125 to 1325$)$ \\
\hline High $(=4)$ & $1.047^{*}$ & $(1.025-1.070)$ & $2041.82^{\ddagger}$ & (1097 to 2986$)$ \\
\hline Very high $(=5)$ & $1.105^{*}$ & $(1.062-1.150)$ & $4528.72^{*}$ & (2664 to 6394$)$ \\
\hline \multicolumn{5}{|c|}{ Distance to hospital (miles) } \\
\hline Short (0 to 15$)$ & Ref & & & \\
\hline Medium (16 to 75 ) & 0.994 & $(0.983-1.006)$ & -249.12 & $(-746$ to 248$)$ \\
\hline Long $(>75)$ & 0.989 & $(0.975-1.003)$ & -483.68 & $(-1101$ to 134$)$ \\
\hline \multicolumn{5}{|l|}{ Percent nonwhite } \\
\hline Quartile1 $(<0.02)$ & Ref & & & \\
\hline Quartile $2(0.02-0.06)$ & 1.002 & $(0.989-1.015)$ & 81.27 & $(-495$ to 658$)$ \\
\hline Quartile 3 (0.07-0.13) & 1.000 & $(0.986-1.015)$ & 17.29 & $(-617$ to 651$)$ \\
\hline Quartile $4(>0.13)$ & 0.991 & $(0.976-1.006)$ & -393.97 & $(-1068$ to 280$)$ \\
\hline \multicolumn{5}{|l|}{ Percent below FPL } \\
\hline Quartile $1(<0.08)$ & Ref & & & \\
\hline Quartile $2(0.08-0.13)$ & 0.990 & $(0.976-1.005)$ & -420.48 & $(-1056$ to 215$)$ \\
\hline Quartile 3 (0.14-0.19) & 0.988 & $(0.975-1.001)$ & -529.23 & $(-1102$ to 43$)$ \\
\hline Quartile $4(>0.19)$ & 0.999 & $(0.984-1.014)$ & -50.50 & $(-719$ to 618$)$ \\
\hline \multicolumn{5}{|l|}{ Hospital characteristics } \\
\hline \multicolumn{5}{|l|}{ Hospital location } \\
\hline Nonurban & Ref & & & \\
\hline Urban & $1.287^{\ddagger}$ & $(1.165-1.422)$ & $9948.95^{\star}$ & $(6271$ to 13,627$)$ \\
\hline
\end{tabular}


Table 4. continued

\begin{tabular}{lllll}
\hline Variable & Rate ratio & $95 \%$ CI & Marginal effects (in USD) & $95 \%$ CI \\
\hline Hospital case mix & & & & \\
$\quad$ Low $(<0.1)$ & 1.000 & $(0.958-1.035)$ & -204.07 & $(-1950$ to 1542$)$ \\
$\quad$ Moderate $(0.1-0.6)$ & 0.995 & $(0.891-0.950)$ & $-3620.54^{\ddagger}$ & $(-5021$ to -2220$)$ \\
High $(>0.6)$ & $0.920^{\ddagger}$ & & & \\
Hospital size (discharges) & & & 1059.35 & $(-420$ to 2538) \\
$\quad$ Small $(<3000)$ & 1.000 & $(0.990-1.061)$ & $3495.30 *$ & $(309$ to 6682) \\
Medium (3000 to 6000) & 1.025 & $(1.009-1.161)$ & \\
Large $(>6000)$ & $1.082^{*}$ &
\end{tabular}

${ }^{*} \mathrm{p}<0.05 ;{ }^{*} \mathrm{p}<0.001 ;$ Ref $=$ reference category; FPL = Federal Poverty Level; TJA = total joint arthroplasty.

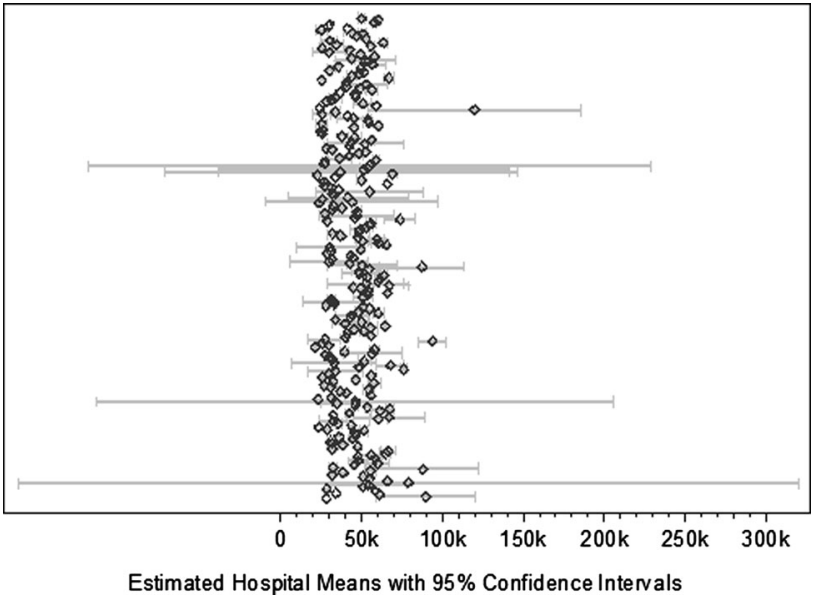

Fig. 2 The estimated means for 90-day episode payments $(\mathrm{k}=$ thousands) among hospitals are shown.

services. This finding can be attributed to our relatively younger and healthier population who were initially discharged home (with or without home health services) after surgery. Our findings are consistent with those of previous studies on episode-of-care payments, which suggests that whereas the index inpatient admission contributes a relatively smaller proportion of total episode-of-care cost component in the Medicare population, it contributes a much-larger proportion of total payments in commercially insured populations $[28,39]$. Thus, the inpatient component of episode-of-care payments remains an important driver of variation in TJA in such populations and will continue to be an essential target for cost containment and the best delivery-improvement strategies.

\section{Procedure-level and Patient-level Factors}

Procedure-characterizing factors were a major source of variation in payments among patients in our sample. Procedure and patient factors associated with higher 90-day mean payments were the index length of stay, higher patient-morbidity burden, any 90-day unplanned readmission, and having a home health versus routine discharge. Previous studies on episode-of-care payments for primary TJA have shown factors such as length of stay, patient comorbidities, type of discharge destination, and readmission rates as drivers of TJA payments [5, 25, 27], and our study results underscore these findings. Clearly, too many 90-day readmissions to an inpatient facility during the postdischarge period will chip away at hospital margins, and future studies that look at factors and interventions that alleviate 90-day readmissions will be useful for hospitals practicing in bundled environments.

Notably, we obtained information regarding the prevalence of computer-assisted surgery in our population and its association with higher payments. Many payers have classified computer-assisted surgery as medically unnecessary or investigational [4, 24, 34] (eg, Blue Cross Blue Shield of Texas requires prior authorization for the use of computerassisted surgery for billing purposes). The final verdict regarding the effectiveness of computer-assisted surgery is uncertain. Some studies have found computer-assisted surgery to be cost-effective in TKA [26] and beneficial in THA [32, 35], whereas others have found no discernible short-term, clinical benefit in TKA or THA [6, 7, 12, 23]. In the cost-reductive environment of the current healthcare system, in the absence of clinical and economic benefits to support the effectiveness of computer-assisted surgery in TJA, the entire additional cost burden of computer-assisted surgery might eventually shift toward the hospital and patients. However, the rapidly changing landscape of technology might yet find computer-assisted surgery to be useful in high-complexity joint surgery.

\section{Hospital-level Factors}

We confirmed that there was high variation in TJA episode payments owing to the nested structure of our data. While 
procedure characteristics were the major source of variation in payments among patients, hospital characteristics were the major source of variation for differences in payments among hospitals. Expanding on a previous study [10], we found that larger hospital size and urban location were associated with higher estimated payments among TJAs while higher case mix was associated with lower payments. The difference in estimated payments between urban and rural hospitals was driven by the inpatient component in our sample, and most likely reflects regional variation in pricing (eg, implant costs) [1, 41]. Hospital size and case-mix differences possibly reflect changes in negotiating leverage between the insurer and hospitals resulting from changes in hospital market power, which has been shown to be positively associated with size and negatively associated with higher hospital case mix [1, 30, 41]. Future research can add measures of competition, such as the Herfindahl-Hirschman Index [37], to measure the effect of market forces on hospital payments.

Overall, the results of our study can inform policymakers, hospitals, and payers considering a shift toward bundled payments in TJA by providing valuable insights in factors associated with higher payments and higher variation in payments for 90-day episodes of care in patients younger than 65 years. Large variation in payments suggests system inefficiencies that may be suitable for the implementation of episode-based bundling. Improved efficiency may be achieved through improved care coordination and standardization of care pathways [16] or through the examination of physician, vendor, and payer relationships for negotiating prices [41]. Future studies also should examine the role of other hospital characteristics such as organizational structure, staffing, physician arrangements, and type of services (such as remote monitoring of discharged patients) when assessing episode payments.

\section{References}

1. Berenson RA, Hoagland GW, Dranove D, Ginsberg P, Glied SA, Goldsmith J, Kocher B, Kramer WE, Levy R, McKeever DP, Pitts KB, Richman BD, Robinson JC, Roosevelt J, Rowe JW, Wolter N. Addressing Pricing Power in Health Care Markets: Principles and Policy Options to Strengthen and Shape Markets. 2015. Available at: https://www.nasi.org/research/2015/ addressing-pricing-power-health-care-markets-principles-poli. Accessed December 2, 2016.

2. Berkowitz SA, Traore CY, Singer DE, Atlas SJ. Evaluating areabased socioeconomic status indicators for monitoring disparities within health care systems: results from a primary care network. Health Serv Res. 2015;50:398-417.

3. Blue Cross Blue Shield Association. The Health of America. A Study of Cost Variations for Knee and Hip Replacement Surgeries in the U.S. Available at: https://www.bcbs.com/the-health- of-america/reports/study-cost-variations-knee-and-hip-replacementsurgeries-us. Accessed December 11, 2016.

4. Blue Cross Blue Shield of North Carolina. Corporate Medical Policy. Computer Assisted Surgical Navigational Orthopedic Procedures. Available at: http://www.bcbsnc.com/assets/services/ public/pdfs/medicalpolicy/computer_assisted_surgical_navigational_ orthopedic_procedures.pdf. Accessed June 21, 2017.

5. Bozic KJ, Ward L, Vail TP, Maze M. Bundled payments in total joint arthroplasty: targeting opportunities for quality improvement and cost reduction. Clin Orthop Relat Res. 2014;472:188-193.

6. Burnett RS, Barrack RL. Computer-assisted total knee arthroplasty is currently of no proven clinical benefit: a systematic review. Clin Orthop Relat Res. 2013;471:264-276.

7. Cheng T, Pan XY, Mao X, Zhang GY, Zhang XL. Little clinical advantage of computer-assisted navigation over conventional instrumentation in primary total knee arthroplasty at early followup. Knee. 2012;19:237-245.

8. CMS.gov. Centers for Medicare \& Medicaid Services. Comprehensive Care for Joint Replacement Model. Available at: https:// innovation.cms.gov/initiatives/cjr. Accessed February 11, 2016.

9. Courtney PM, Huddleston JI, Iorio R, Markel DC. Socioeconomic risk adjustment models for reimbursement are necessary in primary total joint arthroplasty. $J$ Arthroplasty. 2017;32:1-5.

10. Cram P, Ravi B, Vaughan-Sarrazin MS, Lu X, Li Y, Hawker G. What drives variation in episode-of-care payments for primary TKA? An analysis of Medicare administrative data. Clin Orthop Relat Res. 2015;473:3337-3347.

11. Deb P, Manning WG, Norton EC. Modeling Health Care Costs and Counts. iHEA World Congress in Sydney, Australia, 2013. Available at: http://docplayer.net/10668646-Modeling-healthcare-costs-and-counts.html. Accessed June 29, 2017.

12. Desai AS, Dramis A, Kendoff D, Board TN. Critical review of the current practice for computer-assisted navigation in total knee replacement surgery: cost-effectiveness and clinical outcome. Curr Rev Musculoskelet Med. 2011;4:11-15.

13. Dickinson LM, Basu A. Multilevel modeling and practice-based research. Ann Fam Med. 2005;3(suppl 1):S52-S60.

14. Doran JP, Zabinski SJ. Bundled payment initiatives for Medicare and non-Medicare total joint arthroplasty patients at a community hospital: bundles in the real world. J Arthroplasty. 2015;30:353355.

15. Dowsey MM, Nikpour M, Choong PF. Outcomes following large joint arthroplasty: does socio-economic status matter? BMC Musculoskelet Disord. 2014;15:148.

16. Froemke CC, Wang L, DeHart ML, Williamson RK, Ko LM, Duwelius PJ. Standardizing care and improving quality under a bundled payment initiative for total joint arthroplasty. J Arthroplasty. 2015;30:1676-1682.

17. Froimson MI, Rana A, White RE, Marshall A, Schutzer SF, Healy WL, Naas P, Daubert G, Iorio R, Parsley B. Bundled payments for care improvement initiative: the next evolution of payment formulations: AAHKS Bundled Payment Task Force. $J$ Arthroplasty. 2013;28:157-165.

18. John Hopkins Bloomberg School of Public Health. The Johns Hopkins $\mathrm{ACG}^{\mathbb{R}}$ System Technical Reference Guide Version 10.0. Available at: https://www.hopkinsacg.org/document/acgsystem-version-10-0-technical-reference-guide/. Accessed June 29, 2017.

19. Kurtz SM, Lau E, Ong K, Zhao K, Kelly M, Bozic KJ. Future young patient demand for primary and revision joint replacement: national projections from 2010 to 2030. Clin Orthop Relat Res. 2009;467:2606-2612.

20. Manning WG, Basu A, Mullahy J. Generalized modeling approaches to risk adjustment of skewed outcomes data. $J$ Health Econ. 2005;24:465-488. 
21. Manning WG, Mullahy J. Estimating log models: to transform or not to transform? J Health Econ. 2001;20:461-494.

22. Mechanic RE. Mandatory Medicare bundled payment: is it ready for prime time? N Engl J Med. 2015;373:1291-1293.

23. Medical Advisory Secretariat, Health Quality Ontario. Computerassisted hip and knee arthroplasty: navigation and active robotic systems: an evidence-based analysis. Ont Health Technol Assess Ser. 2004;4:1-39. Available at: https://www.ncbi.nlm.nih.gov/ pmc/articles/PMC3387774/. Accessed June 29, 2017.

24. Medical Criteria Committee. Moda Health Medical Necessity Criteria Subject: Computer Assisted Navigation for Musculoskeletal Procedures. Available at: https://www.modahealth. com/pdfs/med_criteria/ComputerAssistedNavigation.pdf. Accessed June 21, 2017.

25. Nichols CI, Vose JG. Clinical outcomes and costs within 90 days of primary or revision total joint arthroplasty. J Arthroplasty. 2016;31:1400-1406.e3.

26. Novak EJ, Silverstein MD, Bozic KJ. The cost-effectiveness of computer-assisted navigation in total knee arthroplasty. J Bone Joint Surg Am. 2007;89:2389-2397.

27. Ramos NL, Wang EL, Karia RJ, Hutzler LH, Lajam CM, Bosco JA. Correlation between physician specific discharge costs, LOS, and 30-day readmission rates: an analysis of 1,831 cases. $J$ Arthroplasty. 2014;29:1717-1722.

28. Rastogi A, de Brantes F, Slusarz J, Tompkins C. Analysis of Medicare and Commercial Insurer-Paid Total Knee Replacement Reveals Opportunities for Cost Reduction. Available at: http:// www.rwjf.org/en/library/research/2010/01/analysis-of-medicareand-commercial-insurer-paid-total-knee-repl.html. Accessed November 28, 2014.

29. Raudenbush SW, Bryk AS. Hierarchical Linear Models: Applications and Data Analysis Methods. Thousand Oaks, CA: Sage Publications; 2002.

30. Robinson JC. Hospital market concentration, pricing, and profitability in orthopedic surgery and interventional cardiology. Am J Manag Care. 2011;17:e241-248.

31. Slover J, Zuckerman JD. Increasing use of total knee replacement and revision surgery. JAMA. 2012;308:1266-1268.
32. Sugano N. Computer-assisted orthopaedic surgery and robotic surgery in total hip arthroplasty. Clin Orthop Surg. 2013;5:1-9.

33. Tomek IM, Sabel AL, Froimson MI, Muschler G, Jevsevar DS, Koenig KM, Lewallen DG, Naessens JM, Savitz LA, Westrich JL, Weeks WB, Weinstein JN. A collaborative of leading health systems finds wide variations in total knee replacement delivery and takes steps to improve value. Health Aff (Millwood). 2012;31:1329-1338.

34. Tufts Health Plan. Medical Necessity Guidelines: Noncovered Investigational Services. Available at: https://tuftshealthplan. com/documents/providers/guidelines/medical-necessity-guidelines/ noncovered-investigational-services-me. Accessed June 21, 2017.

35. Ulrich SD, Bonutti PM, Seyler TM, Marker DR, Jones LC, Mont MA. Outcomes-based evaluations supporting computer-assisted surgery and minimally invasive surgery for total hip arthroplasty. Expert Rev Med Devices. 2007;4:873-883.

36. United States Census Bureau. American Community Survey (ACS). Available at: https://www.census.gov/programs-surveys/ acs/. Accessed November 3, 2014.

37. US Department of Justice. Herfindahl-Hirschman Index. Available at: https://www.justice.gov/atr/herfindahl-hirschman-index. Accessed June 22, 2017.

38. USDA. United States Department of Agriculture. Rural-Urban Continuum Codes. Available at: http://www.ers.usda.gov/dataproducts/rural-urban-continuum-codes.aspx. Accessed April 9, 2016.

39. White C, Reschovsky JD, Bond AM. National Institute for Health Care Reform. Research Brief Number 14. February 2014. Inpatient Hospital Prices Drive Spending Variation for Episodes of Care for Privately Insured Patients. Available at: http://www.nihcr.org/ Episode-Spending-Variation. Accessed November 28, 2014.

40. Whitman E. Divided over bundled payments. Modern Healthcare. September 28, 2016. Available at: http://www.modernhealthcare. com/article/20160928/NEWS/160929877/divided-over-bundledpayments. Accessed October 7, 2016.

41. Wilson NA, Schneller ES, Montgomery K, Bozic KJ. Hip and knee implants: current trends and policy considerations. Health Aff (Millwood). 2008;27:1587-1598. 